\title{
Micotoxinas e alimentos: implicações na saúde humana e animal
}

\author{
(Mycotoxins and food: implications in animal and human health)
}

\author{
Eulógio Carlos Queiroz de Carvalho
}

\section{Resumo}

Este artigo aborda os fatores que favorecem a contaminação dos alimentos humanos e animais por fungos (mofos), tanto pela via direta, quando o próprio alimento serve como substrato para o crescimento de espécies toxigênicas, quanto pela via indireta. As condições para a ocorrência, diagnóstico e controle das micotoxicoses são relatadas. Nos animais de açougue, os efeitos de ordem econômica, sanitária e comercial foram referidos. As micotoxicoses humanas e animais, suas características principais, os agentes com os respectivos substratos e micotoxinas envolvidos foram tabulados. Por fim, o autor revela o propósito de que esta temática, a micotoxicologia, ocupe o espaço que lhe cabe, como um dos mais importantes capítulos da patologia toxicológica atual, ainda relegado.

Palavras chave: micotoxinas, micotoxicoses, alimentos, saúde pública, saúde animal.

\section{Introdução}

Cada vez mais, os metabólicos de fungos, tóxicos ou não, ampliam seu lugar no cenário médico mundial; possuindo propriedades antibióticas, anabolizantes, similestrogênicas, carcinogênicas, mutagênicas, teratogênicas e outras, ao ponto das micotoxinas serem consideradas "poluentes ambientais", quando através da ingestão, inalação e contato direto, produzem as micotoxicoses (Hayes, 1980).

Em climas tropicais e subtropicais como o nosso, que favorecem o crescimento dos fungos e ainda pelos efeitos advindos das estocagens de grandes quantidades de alimentos, principalmente grãos oleaginosos em depósitos inadequados (úmidos e quentes), são freqüentes os acidentes pela ingestão de alimentos mofados atingindo o homem e principalmente os animais, quando, no falso juízo de um aproveitamento condicional do alimento alterado organolepticamente, este é transformado em ração animal.
Dai serem enormes os prejuízos de ordem econômica, sanitária e comercial, oriundos respectivamente, da deterioração (mofo), perda de produtos vegetais e queda na produção de alimentos de origem animal (interferindo no ganho de peso, índices de crescimento e fertilidade, produção de ovos, lã, etc.), da exposição freqüente às micotoxinas com efeitos sobre a saúde animal e humana e das restrições comerciais para exportação de grãos.

A ocorrência natural das micotoxinas na cadeia alimentar humana e animal pode se dar direta e indiretamente. $\mathrm{Na}$ contaminação direta, é o próprio alimento de origem vegetal ou animal, o substrato para o crescimento do fungo toxigênico. A maioria deles, uns mais e outros menos, pode crescer em diferentes estágios da produção, beneficiamento, transporte e/ou armazenagem dos alimentos.

Ao contrário, a contaminação indireta ocorre quando ingredientes do alimento recebem a micotoxina.

As vias possíveis de contaminação por micotoxinas de alimentos humanos e animais são apresentadas no Quadro 1.

As micotoxinas são produzidas por fungos, quando estes atingem seu crescimento máximo. Como resultado, podem permanecer no alimento por anos, mesmo após a morte dos fungos produtores (mofos). Numa ocasião, grãos estocados por 12 anos ainda continham aflatoxina, potente metabólico, típico dos Aspergillus flavus e A. parasiticus, provavelmente formada após o armazenamento (Hayes, 1980).

A ocorrência simultânea de micotoxinas é freqüente e deve ser sempre suspeitada em casos eventuais de micotoxicoses. Nestes, a toxicidade e os sinais clínicos guardadas as proporções da contaminação e a espécie animal envolvida, podem ser complexos e diversos. Aspectos desta interação Têm sido investigados em suínos e aves (Huff et al. 1988).

As micotoxicoses já foram consideradas como "doenças da omissão" (Forgacs, 1962). E é realmente surpreendente que metabólicos tóxicos de fungos, que são uma ameaça à saúde, tenham sido negligenciados por tanto tem-

*Titular em Patologia Veterinária da Universidade Federal Fluminense; Pesquisador do CNPq.

Rua Vital Brazil Filho, 64 - Vital Brazil - Niterói - RJ - Brasil CEP 24230-340 
Quadro 1: Vias de contaminação de alimentos humanos e animais por micotoxinas.

\begin{tabular}{|l|}
\hline ALIMENTOS MOFADOS \\
-Produtos agrícolas \\
-cereais \\
-sementes oleaginosas \\
-frutas \\
-legumes \\
-Alimento animal composto \\
RESÍDUOS EM TECIDOS E PRODUTOS ANIMAIS \\
-Leite \\
-Derivados \\
-Fermentação oriental \\
ALIMENTOS MOFADOS AMADURECIDOS \\
-Queijo \\
-Produtos cárneos fermentados \\
-Fermentação oriental \\
FERMENTOS (PRODUTOS FERMENTADOS) \\
-Proteínas microbianas \\
-Enzimas \\
-Aditivos alimentares (ex.: vitaminas)
\end{tabular}

FONTE: JARVIS.1976 (adaptada)

po, quando outros, não tóxicos ou freqüentemente detectados em doses atóxicas, tiveram seus estudos estimulados, como é o caso daqueles com propriedades antibióticas.

Nos anos 60, no Reino Unido, a descoberta dos efeitos da aflatoxina produzindo doença hepática aguda em perus, patos (filhotes) e outras aves e, em seguida, a demonstração de seu efeito carcinogênico em fígado de animais de laboratório, foi responsável pelo impulso ao estudo das micotoxinas, onde a resposta a uma importante indagação corrente na época, surgiu: as micotoxinas estão envolvidas também na saúde humana? (Hayes, 1980; Patterson, 1983).

Neste surto de aflatoxicose, 100.000 aves morreram em conseqüência da presença da micotoxina na ração (farelo de amendoim) importada do Brasil, levantando sérias restrições ao comércio exterior brasileiro (Rosa, 1985).

\section{Doenças Micotóxicas Humanas}

A susceptibilidade mais exata e especifica do homem às diferentes micotoxinas tem sido difícil de determinar, já que a maior parte dos conhecimentos sobre as mesmas, tem advindo de observações de casos experimentais e naturais em animais. Estas investigações têm demonstrado variações nos efeitos biológicos das micotoxinas, considerando-se a espécie animal, tornando-as de valor limitado na extrapolação para o homem. Estas variações se devem à diversidade das estruturas químicas das micotoxinas e no fato de que introduzidas no organismo dos animais superiores são influenciadas pela raça, sexo, idade, fatores ambientais, condições nutricionais e interação com outras substâncias químicas (Hayes, 1980). No entanto, é possível uma estimativa da susceptibilidade do homem se, apurados os níveis de contaminação do seu alimento, forem ob- servados o tipo e a gravidade de doenças associadas ao consumo do mesmo (Hayes, 1980; Hadidane et al. 1985).

Na Tabela 1 estão relacionadas doenças humanas produzidas por micotoxinas, com comprovação analítica ou sugeridas epidemiologicamente.

A contaminação por micotoxinas está geralmente associada a cereais com alterações organolépticas, os quais não são usualmente utilizados para consumo humano. As principais micotoxinas detectadas em cereais, mais regularmente, têm sido a aflatoxina, sterigmatocistina, ocratoxina A, zeralenona, toxina T-2 e vomitoxina (Stoloff, 1976).

\section{Doenças Micotóxicas Animais}

Cerca de 100 espécies de fungos toxigênicos são conhecidas. Trinta de suas micotoxinas, ocorrendo naturalmente são excepcionalmente importantes em animais como bovinos produtores de leite e de carne, suínos e aves, devido a enorme utilização de ração concentrada/estocada nestas criações. As perdas econômicas devido às micotoxinas são comparadas a um "iceberg" onde se observa somente a ponta representada pelo efeito dramático agudo. Nestes casos os animais que absorvem grande quantidade de toxina, apresentam um quadro agudo que pode ser fatal. No entanto, pela exposição inadvertida e prolongada a baixas doses, a grande maioria dos casos tem caráter subclínico, que pode levar a doença primária crônica e/ou a doenças micotóxicas secundárias.

Assim, as micotoxicoses são distribuídas, de acordo com Pier et al. (1980), em 3 categorias:

\section{1 - Micotoxicose Aguda Primária}

Manifesta-se quando, considerando-se a sensibilidade, os indivíduos consomem doses de moderadas a altas de micotoxinas. Nestes casos, um quadro anátomo-clínico agudo e específico pode incluir sinais e sintomas como hemorragias, hepatite, nefrite, necrose de mucosas digestivas e morte, dependendo da susceptibilidade da espécie, das condições individuais, do organotropismo e interação ou não com outras micotoxinas.

As micotoxicoses agudas importantes para animais de produção (mamíteros e aves) estão listadas na Tabela 2.

\section{2 - Micotoxicose Crônica Primária}

Ocorre quando os níveis de micotoxinas consumidos são de moderados a baixos e se manifesta em quadros representados por redução da eficiência reprodutiva, do ganho de peso e da taxa de crescimento. Em geral, tais efeitos muitas vezes ocorrem sem a caraterização clínica da micotoxicose aguda primária. Além disto, em muitos casos estes efeitos somente serão detectados quando cuidadosamente observados, inclusive através da presença de outras doenças superpostas (oportunistas) e deficiências nutricionais.

\section{3 - Doenças Micotóxicas Secundárias}

Elas resultam da ação de baixos níveis de micotoxinas, incapazes de causar uma micotoxicose evidente, porém, 
Tabela 1 - Micotoxicoses humanas de comprovação analítica ou sugeridas epidemiologicamente*

\begin{tabular}{|c|c|c|c|c|}
\hline Doença & Substrato & Agente & Mixotoxina & Sinais/Lesões \\
\hline $\begin{array}{l}\text {-Aleucia Tóxica Alimentar } \\
\text { (ATA na angina séptica) }\end{array}$ & Cereais & Fusarium sporotrichoides & $\begin{array}{l}\text { Desconhecida } \\
\text { (tricoteceno) }\end{array}$ & $\begin{array}{l}\text { Dermatite, estomatite, necrose linfóide e } \\
\text { leucopenia }\end{array}$ \\
\hline -Dendrodoquiotoxicose & $\begin{array}{l}\text { Forragem (contato } \\
\text { cutâneo, inalação) }\end{array}$ & $\begin{array}{l}\text { Dendrodochim toxicum } \\
\text { (Myrothecium verrucaria } \\
\text { M. roridum) }\end{array}$ & $\begin{array}{l}\text { Verrucarinas } \\
\text { Roridinas }\end{array}$ & $\begin{array}{l}\text { Diarréia, gastroenterocolite hemorrágica, dis- } \\
\text { túrbios respiratórios e ulceração intestinal }\end{array}$ \\
\hline -Estaquibotriotoxicose & $\begin{array}{l}\text { Feno, cereais, for- } \\
\text { ragens (contato cu- } \\
\text { tâneo, inalação) }\end{array}$ & Stachybotrye atra & $\begin{array}{l}\text { Satratoxinas } \\
\text { (Tricotecenos) }\end{array}$ & $\begin{array}{l}\text { Estomatite, ulceração e anorexia: hemorragi- } \\
\text { as extensas em alguns órgãos; inflamação e } \\
\text { necroses intestinais }\end{array}$ \\
\hline $\begin{array}{l}\text {-Doença de Kashin Beck } \\
\text { "Urov Disease" }\end{array}$ & Cereais & Fusarium sporotrichiella & Desconhecida & $\begin{array}{l}\text { Doença degenerativa crônica das articulações } \\
\text { periféricas e da coluna. Ocorre principalmente } \\
\text { na infância e é endêmica na Sibéria, norte da } \\
\text { China e Coréia }\end{array}$ \\
\hline -Beriberi cardíaco & Arroz & Fusarium sp. & Desconhecida & Miocardiopatia \\
\hline -Ergotismo & Cereais, Centeio & Claviceps purpúrea & $\begin{array}{l}\text { Alcalóides do } \\
\text { Ergot }\end{array}$ & $\begin{array}{l}\text { Agudas: diarréia, vômitos, cólicas, cefaléia, } \\
\text { vertigens, parestesia e convulsões. Subagu- } \\
\text { das/crônicas: vasoconstrição com isquemia e } \\
\text { gangrena periféricas. }\end{array}$ \\
\hline -Nefropatia dos Balkans & Cereais & $\begin{array}{l}\text { Penicillium sp. } \\
\text { Aspergillus sp. }\end{array}$ & $\begin{array}{l}\text { Ocratoxina A, } \\
\text { Citrinna. }\end{array}$ & Nefrite intersticial (doença tubulointersticial) \\
\hline -Síndrome de Reye & Cereais & Aspergillus sp. & Aflatoxina B1 & $\begin{array}{l}\text { "Fígado gordo" e encefalopatia não supurativa } \\
\text { aguda (delírio, espasticidade e postura de } \\
\text { descerebração) }\end{array}$ \\
\hline -Hepatocarcinoma & Amendoim, Cereais, & Aspergillus sp. & Aflatoxinas & Hepatocarcinomas \\
\hline -Podridão Rosa & Aipo & Sclerotinia sclerotiorium & 8-netoxipsoralen & $\begin{array}{l}\text { Lesões dérmicas bolhosas por contato direto } \\
\text { com a micotoxina. Comum em colhedores de } \\
\text { aipo. }\end{array}$ \\
\hline -Onyalai & Painço & Phoma sorghina & Desconhecida & $\begin{array}{l}\text { Trombocitopenia aguda (bolhas hemorrágicas } \\
\text { nas mucosas) }\end{array}$ \\
\hline -Câncer urotelial superior & Cereais & $\begin{array}{l}\text { Aspergillus sp. } \\
\text { Penicillium sp. }\end{array}$ & $\begin{array}{l}\text { Ocratoxina } A \text {, } \\
\text { Citrinina }\end{array}$ & $\begin{array}{l}\text { Tumor malígno de células transicionais urete- } \\
\text { rais e da pelve renal (endêmico nos Balkans, } \\
\text { sudeste da Europa, lugoslávia, Romênia e } \\
\text { Bulgária) }\end{array}$ \\
\hline
\end{tabular}

*(Pier et al., 1980) Adaptada 
Tabela 2 - Micotoxicoses agudas primárias em animais domésticos (mamíferos e aves)*

\begin{tabular}{|c|c|c|c|c|c|}
\hline Micotoxicose & Espécie Animal & Substrato & Agente Etiológico & Micotoxina & Doença Primária \\
\hline Aflatoxicose & $\begin{array}{l}\text { Aves, suinos, bovinos } \\
\text { e caninos }\end{array}$ & $\begin{array}{l}\text { Amendoins, soja, caroço de algodão, } \\
\text { arroz, sorgo, milho e outros cereais }\end{array}$ & $\begin{array}{l}\text { Aspergillus flavus } \\
\text { A. parasiticus }\end{array}$ & Aflatoxina & $\begin{array}{l}\text { Hepatite aguda, doença hemorrágica e } \\
\text { morte }\end{array}$ \\
\hline Ergotismo & $\begin{array}{l}\text { Bovinos, ovinos, suinos } \\
\text { e aves }\end{array}$ & Sementes de muitas gramineas e grãos & Claviceps purpurea & $\begin{array}{l}\text { Alcalóides do } \\
\text { Ergot }\end{array}$ & $\begin{array}{l}\text { Necrose gangrenosa, apreensão nervosa } \\
\text { e falhas reprodutivas }\end{array}$ \\
\hline Eczena Facial & Ovinos e bovinos & Esporos tóxicos em palhadas de pastagem & Pithomyces chartarum & Esporidesmina & Colangiohepatite e fotossensibilidade \\
\hline \multicolumn{6}{|l|}{ Fusariotoxicose } \\
\hline Vomitoxicose & Suinos & Cevada com casca & $\begin{array}{l}\text { Fusarium roseus - estágio } \\
\text { sexual: Giberela zeae e outros fuzarios }\end{array}$ & Vomitoxina & Enterite e emese \\
\hline T2 toxicose & Bovinos, suínos e aves & $\begin{array}{l}\text { Cultivo de cereais deixado no campo } \\
\text { durante o inverno, milho em crescimento } \\
\text { e graminea do gênero Festuca }\end{array}$ & $\begin{array}{l}\text { Fuzarium tricinctum } \\
\text { F. roseum; } F \text {. calmorum } \\
\text { F. equiseti; } F \text {. scirpi e } \\
\text { outros }\end{array}$ & $\begin{array}{l}\text { Toxina T2 } \\
\text { (tricoteceno) }\end{array}$ & Dermonecrose e gastroenterite \\
\hline Por Diacetoxiscirfenol & Suinos & $\begin{array}{l}\text { Cultivo de cereais deixado no campo } \\
\text { durante o inverno, milho em crescimento } \\
\text { e graminea do gênero Festuca }\end{array}$ & $\begin{array}{l}\text { Fuzarium tricinvtum } \\
\text { F. roseum; } F \text {. culmorum; } \\
\text { F. equiseti; F. scirpi e } \\
\text { outros }\end{array}$ & $\begin{array}{l}\text { Diacetoxisciffenol } \\
\text { (tricoteceno) }\end{array}$ & $\begin{array}{l}\text { Necrose oral e gastroentérica e } \\
\text { hemorragias }\end{array}$ \\
\hline Leucoencefalomaiacia & Eqüinos & Milho & Fusarium coniliforme & Desconhecida & Depressão nervosa e incoordenação \\
\hline F2 toxicose (Zeralenona) & Suinos & $\begin{array}{l}\text { Milho mofado, alimentos peletizados, } \\
\text { milho em pó e silagem de milho }\end{array}$ & $\begin{array}{l}\text { Fusarium roseum - estágio } \\
\text { sexual: Giberela zeae }\end{array}$ & $\begin{array}{l}\text { Zeralenona } \\
\text { (tricoteceno) }\end{array}$ & Estrogenismo \\
\hline Nefrotoxicose & Suinos e perús & Cevada, milho e trigo & $\begin{array}{l}\text { Penicillium viridicatum } \\
\text { P. citrinum, Aspergillus } \\
\text { ochraceus e outros }\end{array}$ & $\begin{array}{l}\text { Ocratoxina } \\
\text { Citrinina }\end{array}$ & Nefropatia \\
\hline Incordenação por Paspalum & Bovinos, ovinos e eqüinos & $\begin{array}{l}\text { Sementes de gramíneas do gênero } \\
\text { Paspalum }\end{array}$ & $\begin{array}{l}\text { Claviceps paspali, } \\
\text { C. cincerea }\end{array}$ & $\begin{array}{l}\text { Paspalina, paspalitremos } \\
\text { e tremorigenos }\end{array}$ & Ataxia \\
\hline Eslaframinatoxicose & Bovinos e ovinos & $\begin{array}{l}\text { Leguminosas com a "doença da mancha- } \\
\text { negra", principalmente o trevo vermelho } \\
\text { ingeridas com forragem ou feno }\end{array}$ & Rhizotonia leguminicola & $\begin{array}{l}\text { Eslaframina } \\
\text { (alcalóides) }\end{array}$ & Salivação, diarréia e Poliưria \\
\hline Estaquibotritoxicose & Eqüinos & $\begin{array}{l}\text { Pastagem tóxicas ou ferragens ricas } \\
\text { em fibras }\end{array}$ & Stachybotrye atra & $\begin{array}{l}\text { Satratoxinas } \\
\text { (tricotecenos) }\end{array}$ & $\begin{array}{l}\text { Dermonecrose, gastroenterite e } \\
\text { depressão hematopoiética }\end{array}$ \\
\hline Síndrome de Tremorgenataxia & Bovinos, ovinos e caninos & Alimento mofado & $\begin{array}{l}\text { Penicillium crustosum } \\
\text { P. puberulum, } P \text {. verruculosum, } \\
\text { Aspergillus flavus } \\
\text { A. fumigatus e outros }\end{array}$ & $\begin{array}{l}\text { Penitremos, verruculogeno, } \\
\text { Paxilina, Fumitremórgenos } \\
\text { eaflatremos }\end{array}$ & Fasciculação, ataxia e prostração \\
\hline Intoxicação por trevo doce & $\begin{array}{l}\text { Bovinos, eqüinos, suínos } \\
\text { e ovinos }\end{array}$ & Trevo doce (Melilotus sp.) & $\begin{array}{l}\text { Penicillium sp. } \\
\text { Mucor sp. e Aspergillus sp. }\end{array}$ & Dicumarol & Hemorragias \\
\hline
\end{tabular}

*(Hayes, 1980; Jankovic et al., 1988) Adaptada 
capazes de predispor o hospedeiro a doenças infecciosas através da imunossupressão e da quebra de outros mecanismos de resistência orgânica (depressão da fagocitose, queda na produção de complemento), demonstrados inclusive experimentalmente (Pier et al., 1978). A imunodepressão é implicada com muitas micotoxinas. Com a aflatoxina, este efeito pode se dar a níveis abaixo daqueles necessários para a aflatoxicose clínica. Em doenças micotóxicas secundárias, os sinais/sintomas são aqueles do processo a que o hospedeiro estava predisposto.

Para o diagnóstico das micotoxicoses é imperativa a demonstração de concentrações da toxina no alimento e/ ou de seus resíduos no tecido animal, urina ou leite, e ainda demonstrar as alterações anatomo-clínicas no animal suspeito (Nelson \& Christensen, 1976). Não basta demonstrar espécies toxigênicas de fungos nos alimentos para se caracterizar a micotoxicose. Estes fungos são relativamente ubiquos e para as espécies toxigênicas devem haver condições necessárias para a elaborarão da micotoxina. Por outro lado, o fungo toxigênico pode não estar mais presente no alimento em que produziu a micotoxina (Pier et al., 1980)

É indubitável que, para controlar a contaminação dos alimentos por micotoxinas, a melhor medida é evitar a produção da mesma, através de uma tecnologia de alimentos adequada. Para o controle do crescimento de fungos (mofo) e subseqüente produção de micotoxinas, é importante evitar danos aos grãos durante a colheita, rápido beneficiamento pós-colheita e um perfeito armazenamento.

A micotoxicologia como temática diretamente relacionada aos alimentos, tem-se revelado, nos dias atuais, como um dos mais importantes capítulos da Patologia Toxicológica humana e animal. Entretanto, ainda relegada, merece mais atenção das autoridades sanitárias e dos estudiosos.

\section{Abstract}

\section{Mycotoxins and food: implications in animal and human health}

This paper reports the factors related with the human and animal contamination by fungi (molds), through direct way when food plays the role of the substratum for the growing of toxigenic species or through indirect way. Conditions to the ocurrence, diagnosis and control of the mycotoxicosis are listed. The effects of economical, sanitary and commercial order in the slaughtering animals were referred. Human and animal mycotoxicosis, their main characteristics, the agents with their substratum and mycotoxins were shown in tables. Finally, the author mencioned his purpose that this subject mycotoxicology - can reach in the future its real dimension as the most important chapter of the modern toxicological pathology.

Key words: mycotoxins; mycotoxicosis; food; human health; animal health.

\section{Referências}

FORGACS, J. - Mycotoxicoses: The Neglected Diseases, Feedstuffs, v. 34, p. 124,1962

HADIDANE, R., ROGER-REGNAULT, T.C.; BOUATTOUR, H.; ELLOUZE, F., BACHA, H., CREPPY, E.E. \& DIRHEIMER, G. - Correlation betwen alimentary mycotoxin contamination and specific diseases. Hum. Toxicol. v. 4 , n. 5, p. 491-501, 1985

Hayes, A.W.- Micotoxins: Areview of biological effects and their role in human diseases. Clinical Toxicol v.17, n.45-83, 1980

HUFF, W.E., KUBENA, L.F., HARVEY, R.B. \& DOERR, J.A. - Micotoxin interactions in poultry and swine. J. Anim. Sci., v. 66, n. 9 p. 2351-2355, 1988.

JANKOVIC, S., MARINKOVIC, J. \& RADOVANOVIC, Z. - Survival of the upperurothelial-cancer patients from the balkan nephropathy endemic and nonendemic areas. Eur. Urol. v. 15, p. 39-61, 1988.

JARVIS, B. - Micotoxins in food; in: Microbiology in agriculture, fisheries and food. Washington D.C.: Ed. F. A. Skinner, J.G. Carr, 1976. pp. 251-267.

NELSON, G.H., CHRISTENSEN, C.M.: Mycotoxins and mycotoxicosis. 1 Diagnosing, cases of poisoning, identifying the causative agent, and establishing effective control. Mod. Vet. Pract. p. 529-532, 1976

PATTERSON, P.S.P. Aflatoxicosis in farm animals. Vet. Res. Commun.v. 7 p. $153-140,1983$

PIER, A.C., RICHARD, J.L., CYSEWSKI, S.J. - Implications of mycotoxins in animal disease. J. Am. Vet. Med. Assoc. v. 176 p. 719-724, 1980.

PIER, A.C., RICHARD, J.L., THURSTON, J.R. - The influence of mycotoxins on resistance and immunity, in: Interation of mycotoxins in animal production Washington D.C.: National Academy of Science, 1978, Pl. 56-66.

ROSA, C.A.R. Perfil bioquímico sérico de suínos experimentalmente intoxicados com citrinina. Itaguaí, RJ, 1985. 67p. Tese (mestrado). Universidade Federal Rural do Rio de Janeiro.

STOLOFF, L. Ocurrence of mycotoxins in foods and feeds; in: mycotoxins and other fungal related food problems. Washington, Ed. J. V. Rodricks. Americam Chemical Society, 1976 